\title{
Primary Lung Cancer
} Complicated by Malignant Lymphoma in Two Cases of
Epstein-Barr Virus Infection

\author{
Zentaro Ohno $^{\text {a }}$ Hidetoshi Tamaki $^{\text {a }}$ Takeshi Ohsuga $^{a}$ \\ Hiroyuki Iwata $^{a}$ Norio Yasuda $^{a}$ Yoshio Mori $^{\text {b }}$ \\ Departments of ${ }^{\mathrm{a}}$ Internal Medicine and ${ }^{\mathrm{b}}$ Pathology, Chuno Kosei Hospital, \\ Seki, Japan
}

\section{Key Words}

Double cancer $\cdot$ Lung adenocarcinoma $\cdot$ Diffuse large B-cell-type lymphoma Epstein-Barr virus infection

\begin{abstract}
Background: Double cancer is defined as the co-existence of two pathologically distinct cancers. Double cancer consisting of a lung adenocarcinoma and a malignant lymphoma has seldom been reported in time synchronous cases or prior to cases of primary lung cancer, except in those after treatment for malignant lymphoma.
\end{abstract}

Case Presentation: Case 1 was a 71-year-old woman who was treated at our hospital for chronic hepatitis $\mathrm{C}$, nontuberculous mycobacteria infection, and bronchiectasis. She was diagnosed with a stage IV lung adenocarcinoma (cT1bN2M1b) with a synchronous complicating diffuse large B-cell-type lymphoma. Case 2 was a 62-year-old man who had undergone resection of a stage IB lung adenocarcinoma (pT2aNOMO). Thirty months after the surgery, a diffuse large B-cell-type lymphoma was discovered. In both cases, high antiviral capsid antigen IgG antibody titers were observed.

Conclusion: Epstein-Barr virus may be associated with the incidence of multiple cancers given the pathological evidence from our two double cancer cases.

\section{Introduction}

Lung cancer after treatment for malignant lymphoma has been previously reported $[1,2]$. However, there are few reports of lung cancer prior to complicating malignant lymphoma or their coincidence. Epstein-Barr virus (EBV) was discovered in 1964 when Epstein and Barr isolated the virus from Burkitt's lymphoma. A microRNA study has 
since found EBV infection in other types of lymphoma [3]. At present, however, there is no definitive evidence of EBV playing a causative role in lung cancers [4]. This report describes two cases in which EBV was found in primary lung cancer complicated by a malignant lymphoma.

Written consent for the publication of the two cases was provided by the patients. This study followed the Declaration of Helsinki on Ethical Principles for Medical Research Involving Human Subjects.

\section{Case Presentation}

\section{Case 1}

A 71-year-old woman who was treated at our hospital for chronic hepatitis C, hemoptysis due to bronchiectasis, and nontuberculous mycobacteria infection consulted us about a new symptom of swelling of the left cervical lymph node (CLN). She had received interferon therapy for hepatitis $\mathrm{C}$ at our hospital, and she was a nonsmoker. A whole-body examination was performed immediately. Needle biopsy of the CLN revealed a diffuse large B-cell-type lymphoma (DLBCL), and transbronchial biopsy (TBB) with a bronchofiberscope revealed primary lung cancer (fig. 1). Pathological examination confirmed stage IV adenocarcinoma (cT1bN2M1b). Immunofluorescence assay revealed that EB antiviral capsid antigen (VCA)-IgG titer was high (40 times; standard value: 10 times), while EB VCA-IgM titer was normal (10 times; standard value: 10 times), indicating a previously acquired nonacute infection with EBV.

From the standpoint of prognosis, treatment for lung cancer was prioritized and paclitaxel and bevacizumab with carboplatin were started. However, after the first course of systemic cytotoxic chemotherapy, the regimen was changed to erlotinib because of delayed nadir and recovery, and the patient was discharged.

\section{Case 2}

A 62-year-old man underwent resection of a stage IB lung adenocarcinoma (pT2aN0M0) of the

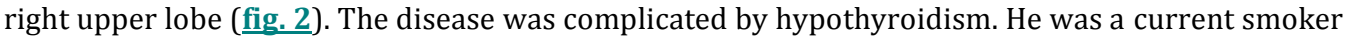
( 40 cigarettes per day $\times 30$ years). Immediately after surgery, the patient was started on tegafur/uracil against brain metastasis, but this was stopped due to a decline in liver function. Thirty months after the surgery, the patient presented with convulsions due to brain metastasis. At this time, his right neck was noted to be swollen and DLBCL was confirmed. Similarly to case 1, EB VCA-IgG titer was high (320 times), while EB VCA-IgM titer was normal. This, again, indicated a previously acquired nonacute infection with EBV. Gamma knife radiosurgery was performed to resect the brain metastatic lesion, and the convulsions were controlled with four courses of systemic chemotherapy with paclitaxel and carboplatin.

\section{Discussion}

Both patients in the presented cases had a lung adenocarcinoma at the apex of the lung and complicating DLBCL involving the CLN. Further, they both had high EB VCAIgG titers. A possible explanation for the clinical course in these two cases is that EBV was reactivated in the patients' immunocompromised state following treatment for lung cancer and became a carcinogen for malignant lymphoma. In support of this speculation, Feng et al. [5] reported that administration of the immunosuppressive drug methotrexate for rheumatoid arthritis will reactivate latent EBV and thereby contribute to methotrexate-associated lymphomas. EBV-negative lymphomas have been reported, and Oyama et al. [6] reported that of a total of 96 patients (age: $>60$ 
years) with B-cell lymphoproliferative disorders, 79 were EBV-positive and the survival rate was poor.

However, in a study using microRNA analysis, Koshiol et al. [4] reported that EBV had only a minor association with the etiology of lung cancer. Thus, whether EBV contributes to the pathogenesis of lung cancer remains unclear. In Japan, there is a higher incidence of non-Hodgkin than Hodgkin lymphoma compared to Western countries. The etiologies of these diseases may differ. Currently, there are no comprehensive theories of the etiology of histological types of cancer.

It is important that oncologists do not overlook the possibility of double cancers, because it is clearly more difficult to treat such cases. Checking for current or past EBV infection has the potential to identify a refractory double cancer at an early stage of the surveillance process. While it is interesting that the location of the double cancer was the same in our two cases, there are too few cases to determine whether this location has any significance. The present cases suggest that over the long term, EBV may be a causative factor for refractory double cancer.

\section{Acknowledgement}

We thank Dr. Yoshinobu Hirose of the Department of Tumor Pathology, Gifu University Graduate School of Medicine, for the pathological diagnosis in case 2.

\section{Disclosure Statement}

All authors declare that they have no conflict of interest. 


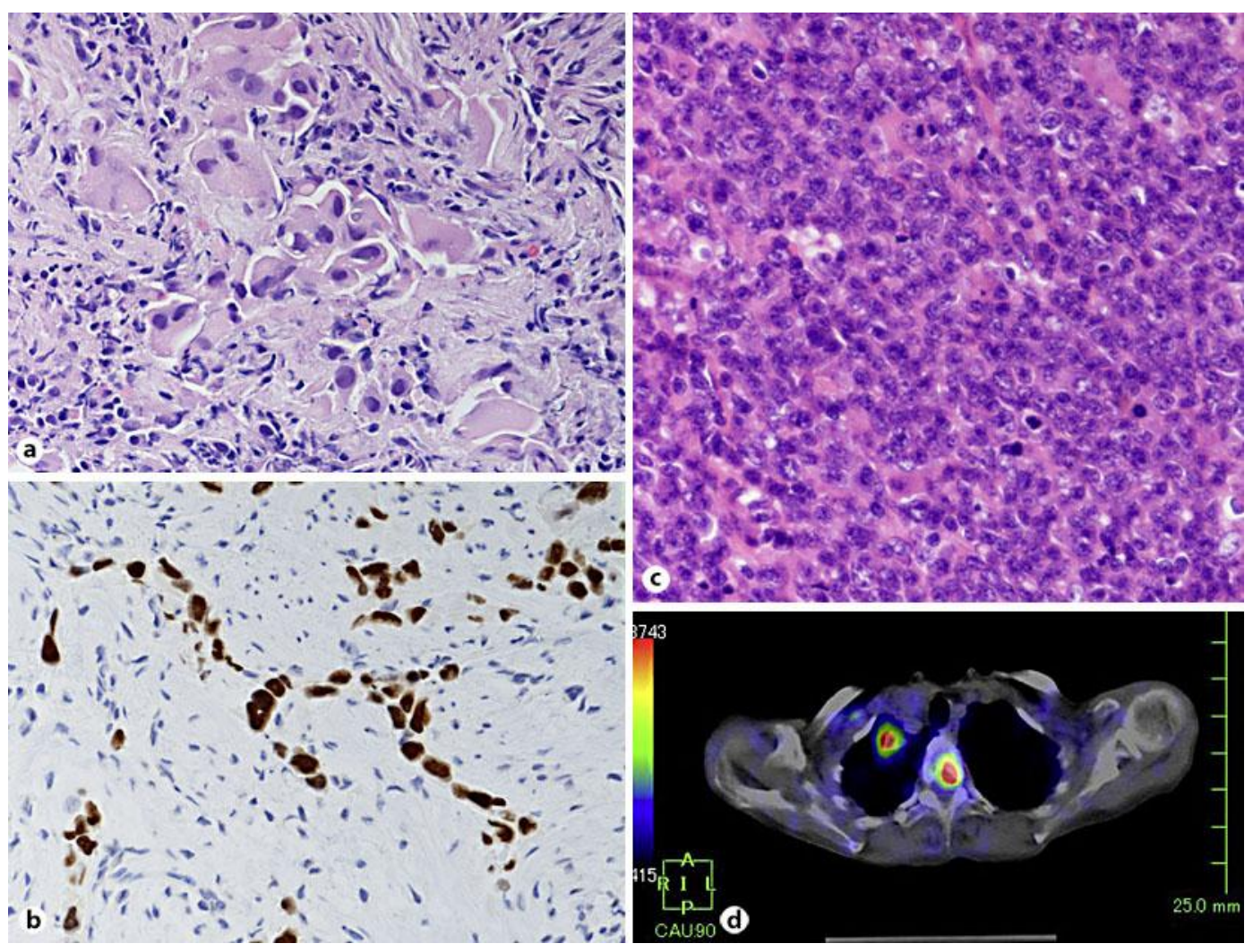

Fig. 1. Pathological and imaging findings in case 1. a TBB specimen from the right lung tumor (HE stain). Pathological diagnosis was adenocarcinoma. b TBB specimen from the right lung tumor. Immunohistochemical stain for thyroid transcription factor-1 was positive, indicating adenocarcinoma. c HE stain of biopsy specimen from the left CLN. Pathological diagnosis was diffuse DLBCL. Large atypical lymphocytes diffusely proliferated. Large nuclei have a coarse nucleoreticulum that includes several small nucleoli. Mitosis is occasionally apparent. $\mathbf{d}$ Fusion image of fluorodeoxyglucose positron emission tomography/computed tomography shows high accumulation of fluorodeoxyglucose in $\mathrm{S}^{3}$ of the right lung and a bone metastatic lesion in the vertebral body. Maximum standard uptake value of the lesion in the right apex of the lung was 4.68 in the early phase and 5.61 in the delayed phase. 


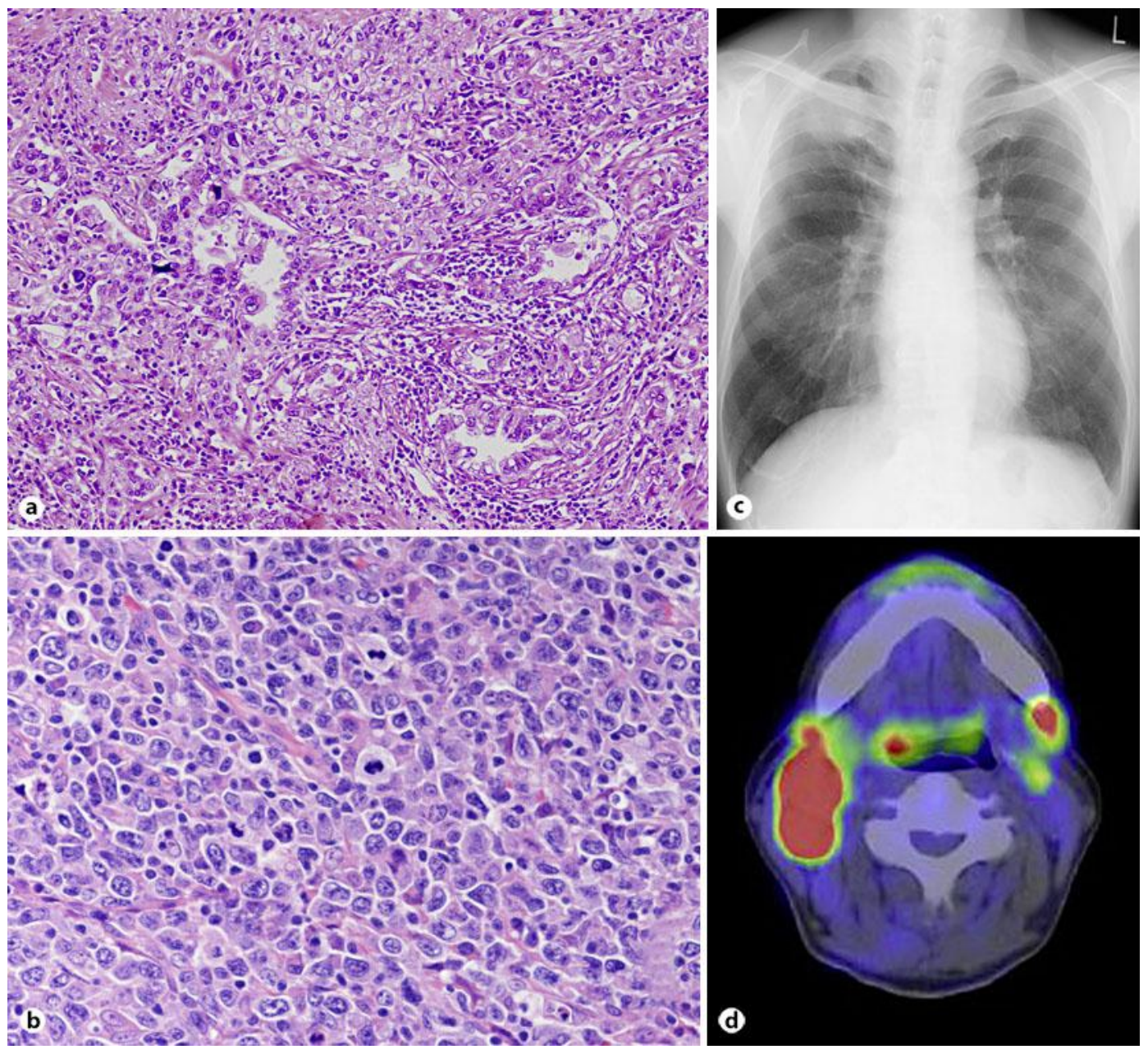

Fig. 2. Pathological and imaging findings in case 2. a Surgically resected specimen from the right upper lobe shows duct-like structures. The pathological diagnosis was low-grade adenocarcinoma. b Preoperative chest X-ray shows a lung tumor in the right upper lung field behind the right clavicle. Silhouette sign is negative. c Diffuse DLBCL in the right CLN. Large atypical lymphocytes have proliferated diffusely in the CLN. Some large nucleoli are apparent against a coarse nucleoreticulum background. Mitosis can be seen in places. $\mathbf{d}$ High accumulation of fluorodeoxyglucose in the right CLN with a maximum standard uptake value of 23.6. 


\section{References}

1 Lorigan P, Radford J, Howell A: Lung cancer after treatment for Hodgkin's lymphoma: a systematic review. Lancet Oncol 2005;6:773-779.

2 Schoenfeld JD, Mauch PM, Das P: Lung malignancies after Hodgkin lymphoma: disease characteristics, detection methods and clinical outcome. Ann Oncol 2011, E-pub ahead of print.

-3 Xia T, O'Hara A, Araujo I: EBV microRNAs in primary lymphomas and targeting of CXCL-11 by ebv-mirBHRF1-3. Cancer Res 2008;68:1436-1442.

-4 Koshiol J, Gulley ML, Zhao Y: Epstein-Barr virus microRNAs and lung cancer. Br J Cancer 2011;105:320326.

5 Feng WH, Cohen JI, Fischer S: Reactivation of latent Epstein-Barr virus by methotrexate: a potential contributor to methotrexate-associated lymphomas. J Natl Cancer Inst 2004;17:1691-1702.

-6 Oyama T, Yamamoto K, Asano N: Age-related EBV-associated B-cell lymphoproliferative disorders constitute a distinct clinicopathologic group: a study of 96 patients. Clin Cancer Res 2007;13:51245132.

Presented in part at the 100th Domestic Conference of The Japan Lung Cancer Society at Nagoya, Japan, on February 18, 2012. 